\title{
Novel USH2A mutations in Japanese Usher syndrome type 2 patients: marked differences in the mutation spectrum between the Japanese and other populations
}

\author{
Hiroshi Nakanishi ${ }^{1,2}$, Masafumi Ohtsubo ${ }^{2}$, Satoshi Iwasaki ${ }^{3}$, Yoshihiro Hotta ${ }^{4}$, Shin-ichi Usami ${ }^{5}$, \\ Kunihiro Mizuta ${ }^{1}$, Hiroyuki Mineta ${ }^{1}$ and Shinsei Minoshima ${ }^{2}$
}

Usher syndrome (USH) is an autosomal recessive disorder characterized by retinitis pigmentosa and hearing loss. USH type 2 (USH2) is the most common type of USH and is frequently caused by mutations in USH2A. In a recent mutation screening of USH2A in Japanese USH2 patients, we identified 11 novel mutations in 10 patients and found the possible frequent mutation c.8559-2A $>$ G in 4 of 10 patients. To obtain a more precise mutation spectrum, we analyzed further nine Japanese patients in this study. We identified nine mutations, of which eight were novel. This result indicates that the mutation spectrum for USH2A among Japanese patients largely differs from Caucasian, Jewish and Palestinian patients. Meanwhile, we did not find the c.8559-2A $>$ G in this study. Haplotype analysis of the c.8559-2G (mutated) alleles using 23 single nucleotide polymorphisms surrounding the mutation revealed an identical haplotype pattern of at least $635 \mathrm{~kb}$ in length, strongly suggesting that the mutation originated from a common ancestor. The fact that all patients carrying c.8559-2A $>$ G came from western Japan suggests that the mutation is mainly distributed in that area; indeed, most of the patients involved in this study came from eastern Japan, which contributed to the absence of c.8559-2A $>\mathbf{G}$.

Journal of Human Genetics (2011) 56, 484-490; doi:10.1038/jhg.2011.45; published online 19 May 2011

Keywords: haplotype; hearing loss; retinitis pigmentosa; Usher syndrome; USH2A

\section{INTRODUCTION}

Usher syndrome (USH) is an autosomal recessive disorder characterized by retinitis pigmentosa (RP) and hearing loss (HL), with or without vestibular dysfunction. ${ }^{1}$ It is the most common cause of combined deafness and blindness in industrialized countries, with a general prevalence of 3.5-6.2 per 100000 live births. ${ }^{2-7}$ The syndrome is clinically and genetically heterogeneous and can be classified into three clinical subtypes on the basis of the severity and progression of $\mathrm{HL}$ and the presence or absence of vestibular dysfunction. ${ }^{8-10}$

USH type 2 (USH2) is characterized by congenital mild-to-severe $\mathrm{HL}$ and a normal vestibular response; it is the most common type and accounts for $>50 \%$ of USH cases. ${ }^{6}$ Three causative genes have been identified: Usher syndrome 2A (USH2A), G-protein coupled receptor 98 (GPR98) and deafness, autosomal recessive 31 (DFNB31). ${ }^{11-14}$ USH2A, which encodes usherin, accounts for $74-90 \%$ of USH2 cases. ${ }^{15-17}$ Usherin is a large protein comprising many functional domains (Figure 1a). ${ }^{11,12}$

Mutation analysis of USH2A for the full-length coding region (exons 1-73) in Caucasian patients has revealed the frequent mutation
p.Glu767fs (c.2299delG) in exon $13 .{ }^{18-21}$ We recently analyzed USH2A in Japanese USH2 patients, in which the p.Glu767fs mutation was not identified, and identified 11 novel mutations in 10 patients, indicating that the mutation spectrum of $U S H 2 A$ in Japanese patients is considerably different from Caucasians. ${ }^{22}$ As 8 of 10 patients had mutations in USH2A, the incidence of mutations in the Japanese was similar to that of Caucasians; furthermore, we found the c.8559$2 \mathrm{~A}>\mathrm{G}$ splicing mutation in 4 of 10 patients. We suggested that c.8559-2A $>\mathrm{G}$ was a possible frequent USH2A mutation among Japanese USH2 patients. In this study, we further performed mutation analysis of $U S H 2 A$ in nine USH2 patients to examine if our previous findings were representative of the Japanese population and to obtain a more precise mutation spectrum.

\section{MATERIALS AND METHODS}

Subjects and diagnosis

Nine unrelated Japanese patients were referred to Hamamatsu University School of Medicine for genetic diagnosis of USH. All these patients were not related to the previously reported patients ${ }^{22}$ and met the following criteria for

${ }^{1}$ Department of Otolaryngology, Hamamatsu University School of Medicine, Hamamatsu, Japan; ${ }^{2}$ Department of Photomedical Genomics, Basic Medical Photonics Laboratory, Medical Photonics Research Center, Hamamatsu University School of Medicine, Hamamatsu, Japan; ${ }^{3}$ Department of Hearing Implant Sciences, Shinshu University School of Medicine, Matsumoto, Japan; ${ }^{4}$ Department of Ophthalmology, Hamamatsu University School of Medicine, Hamamatsu, Japan and ${ }^{5}$ Department of Otorhinolaryngology, Shinshu University School of Medicine, Matsumoto, Japan

Correspondence: Dr S Minoshima, Department of Photomedical Genomics, Basic Medical Photonics Laboratory, Medical Photonics Research Center, Hamamatsu University School of Medicine, 1-20-1 Handayama, Higashi-ku, Hamamatsu 431-3192, Japan.

E-mail: mino@hama-med.ac.jp

Received 1 February 2011; revised 30 March 2011; accepted 1 April 2011; published online 19 May 2011 


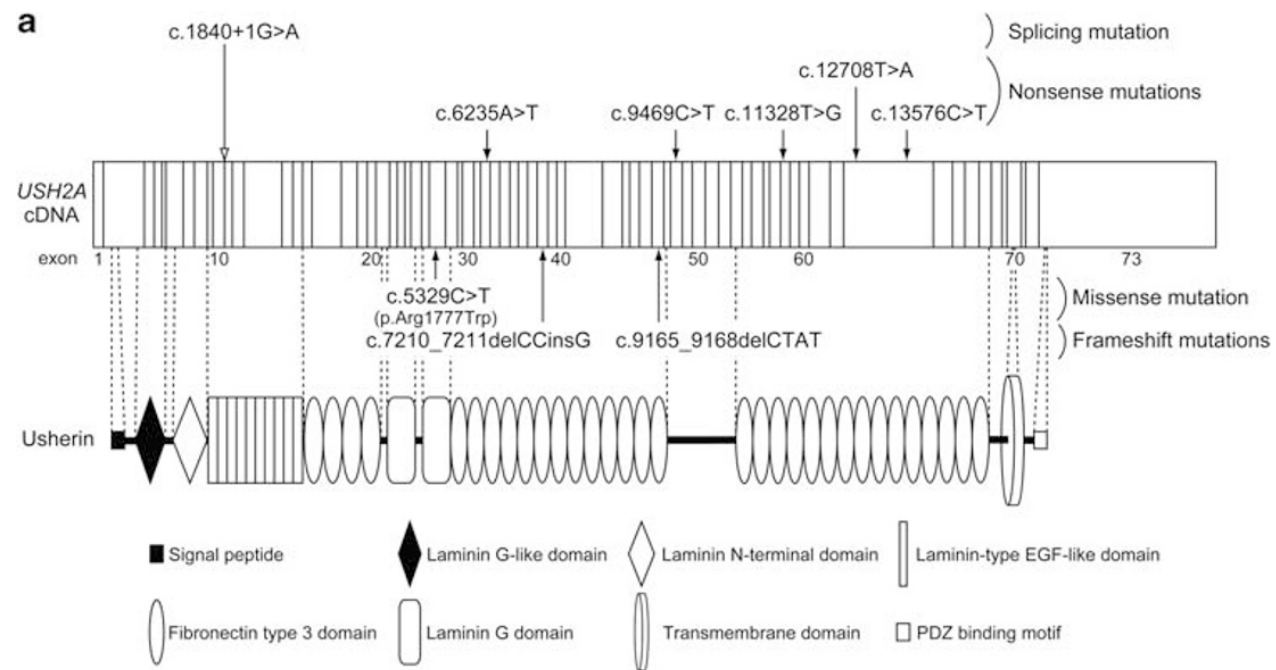

b

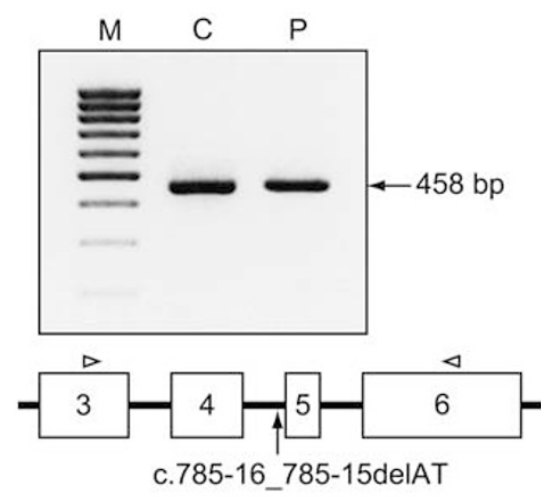

c

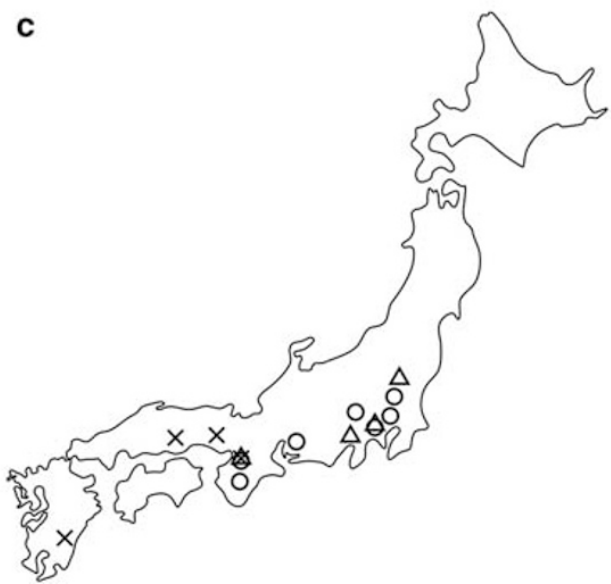

Figure 1 (a) Schematic distribution of mutations identified in USH2A. Upper, USH2A complementary DNA (cDNA) with exon boundaries. Lower, usherin domains encoded by USH2A. All mutations were widely distributed almost throughout the entire USH2A region without any apparent hot spot. The open arrow indicates a mutation in an intron and closed arrows indicate mutations in exons. (b) Products of RT-PCR performed using primers to amplify USH2A CDNA between exons 3 and 6 . Agarose gel electrophoresis of the RT-PCR products revealed a single band of the size predicted from the normal sequence, indicating that the nucleotide change (c.785-16_785-15delAT) had no effect on splicing and was presumably non-pathogenic. PCR was performed using $2 \mu \mathrm{g}$ cDNA (total volume, $20 \mu \mathrm{l}$ ) with 40 cycles. The boxes with a number represent exons. The distance between the exons does not indicate the accurate sizes of the introns. The open arrowheads indicate the PCR primers and the arrow indicates the nucleotide change. M, molecular marker (100-bp ladder); C, control; P, patient. (c) Schematic distribution of the patients in whom at least one mutated allele was detected. Circles indicate patients included in this study, and triangles and crosses indicate patients included in the previous study. In particular, crosses indicate the patients in whom $\mathrm{c} .8559-2 \mathrm{~A}>\mathrm{G}$ was detected.

USH2: RP, congenital mild-to-severe HL and normal vestibular function. ${ }^{8}$ The clinical evaluation of the affected patients consisted of an elicitation of their medical history, and ophthalmological and audiovestibular examinations. The medical history included their place of birth, age at diagnosis of HL, age at onset of night blindness and age at diagnosis of RP. The details of the ophthalmological and audiovestibular examinations were described in a previous report. ${ }^{23}$

A set of 135 control subjects, selected from Japanese individuals with no visual or hearing impairment, was used to access the frequency of nucleotide sequence variations. The Institutional Review Board of Hamamatsu University School of Medicine approved this study, and written informed consent was obtained from all subjects before enrollment.

\section{Mutation analysis}

Genomic DNA was extracted from peripheral lymphocytes with standard procedures. All 73 exons of USH2A and their flanking sequences were amplified by PCR. The PCR products were purified with the Wizard SV Gel and PCR Clean-up System (Promega, Madison, WI, USA) or treated with Exonuclease I and Antarctic Phosphatase (New England Biolabs, Ipswich, MA, USA). Direct sequencing was performed using the BigDye Terminator version 3.1 Cycle Sequencing Kit on an ABI 3100 Autosequencer (Applied Biosystems, Foster City, CA, USA). The PCR primers used for USH2A amplification were described previously. ${ }^{22}$ Using direct sequencing or a restriction enzyme-based assay, we tested the Japanese control chromosomes for the novel mutations identified during the mutation analysis.

\section{In silico analysis for the pathogenicity of a missense mutation}

We used the Sort Intolerant From Tolerant (SIFT) (http://sift.jcvi.org) ${ }^{24}$ and the polymorphism phenotyping (PolyPhen) (http://genetics.bwh.harvard.edu/pph/) ${ }^{25}$ programs to analyze the pathogenicity of a missense mutation. The results from SIFT analysis are given by a probability from $0-1$, where mutations with a probability $<0.05$ are predicted to be 'deleterious', whereas those with a probability $\geqslant 0.05$ are predicted to be 'tolerated.' PolyPhen describes the mutations as 'benign,' 'possibly damaging' or 'probably damaging.' We applied these programs as described by McGee et al. ${ }^{26}$ 


\section{Reverse-transcriptase (RT)-PCR}

We previously showed the significant expression of mRNA in hair roots for seven of nine USH-causing genes (including USH2A); ${ }^{27}$ therefore, we used total RNA extracted from hair roots and performed RT-PCR to examine the effect of a splicing site change on transcript. Novel PCR primers were designed: forward primer, 5'-GCACAGTAAATGGTTTGCAACCTCCAA-'3 located in exon 3; and reverse primer, $5^{\prime}$-AGGATGGGCTTCAGGATTCAACCG-' 3 in exon 6 . The amplification conditions were as follows: denaturation at $94^{\circ} \mathrm{C}$ for $2 \mathrm{~min}, 40$ cycles of $98^{\circ} \mathrm{C}$ for $10 \mathrm{~s}, 60^{\circ} \mathrm{C}$ for $30 \mathrm{~s}$ and $68^{\circ} \mathrm{C}$ for $1 \mathrm{~min}$, and a final extension at $68^{\circ} \mathrm{C}$ for $5 \mathrm{~min}$.

\section{Haplotype analysis}

Haplotype pattern within the region surrounding position c.8559-2, where the 'frequent' Japanese mutation c.8559-2A $>\mathrm{G}$ was found, was analyzed using a set of 23 single nucleotide polymorphisms (SNPs) (10 sites upstream and 13 sites downstream), which were previously described by ourselves or others. ${ }^{15,18,20,22,28,29}$ Haplotype analysis was performed by a direct sequencing method. ${ }^{12,15,22}$

\section{RESULTS}

\section{Mutation analysis}

Mutation analysis of USH2A in the nine unrelated Japanese patients revealed nine probable pathogenic mutations in seven patients (Tables 1 and 2; Figure 1a). Of these, eight mutations were novel (Table 2). The p.Arg1777Trp missense mutation was identified in two patients (C552 and C187), whereas the other mutations were detected in one patient each. The mutations were widely distributed almost throughout the entire region of $U S H 2 A$, without any apparent mutation hot spot (Figure 1a). Two mutations (p.Cys4236X and p.Arg4526X) were found in a homozygous state, of which p.Arg4526X could probably be accounted for by consanguinity (Supplementary Figure 1). In four of the seven patients (C696, C112, C644, and C552), two probable pathogenic alleles were identified and confirmed to be on different chromosomes by using parent or sibling samples (Table 1). For patient C187, segregation analysis could not be performed due to the difficulties in collecting samples from family members. Therefore, we could not confirm whether the two mutations (p.Arg1777Trp and p.Pro2404ValfsX9) were located on different chromosomes. In two patients (C185 and C406), only one mutation was identified; the other mutation remained undetected. None of these nine mutations were found in the Japanese control chromosomes (Table 2).

In the nine mutations identified in this study, seven were of the truncated type, whereas two (c.1840+1G $>$ A and p.Arg1777Trp) were not. The c.1840+1G $>$ A splicing mutation was presumed to be pathogenic because the mutation affected the strictly conserved sequences of a splicing donor site and was not found in 270 Japanese control chromosomes. Its precise effect on splicing was not examined as RT-PCR analysis could not be performed due to the difficulties in collecting hair roots from patient C644. The p.Arg1777Trp missense mutation was also presumed to be pathogenic because the mutation was found in two patients (C552 and C187), but not in 270 Japanese control chromosomes. However, Arg1777 in usherin encoded by USH2A was not evolutionally conserved compared with that encoded by orthologous genes of various vertebrates. In silico analysis for the pathogenicity of this mutation with the SIFT and PolyPhen programs generated a 'tolerated' rating (probability 0.09 ) by SIFT and a 'possibly damaging' rating by PolyPhen, which did not exclude the small possibility that this mutation is non-pathogenic. Thus, further analysis may be necessary to determine the precise nature of the mutation.

In addition to the probable pathogenic mutations listed in Table 2, 36 sequence alterations were identified (Table 3 and Supplementary Table 1). These alterations were predicted to be non-pathogenic for

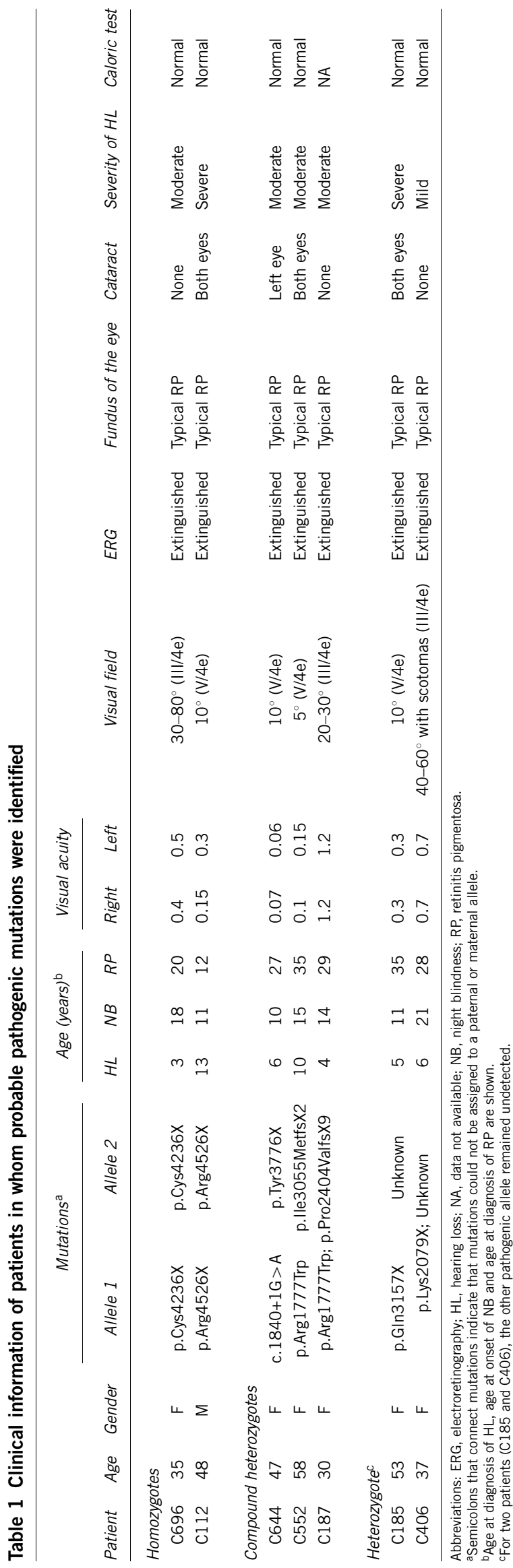


Table 2 Probable pathogenic mutations identified in the Japanese USH2 patients examined in this study

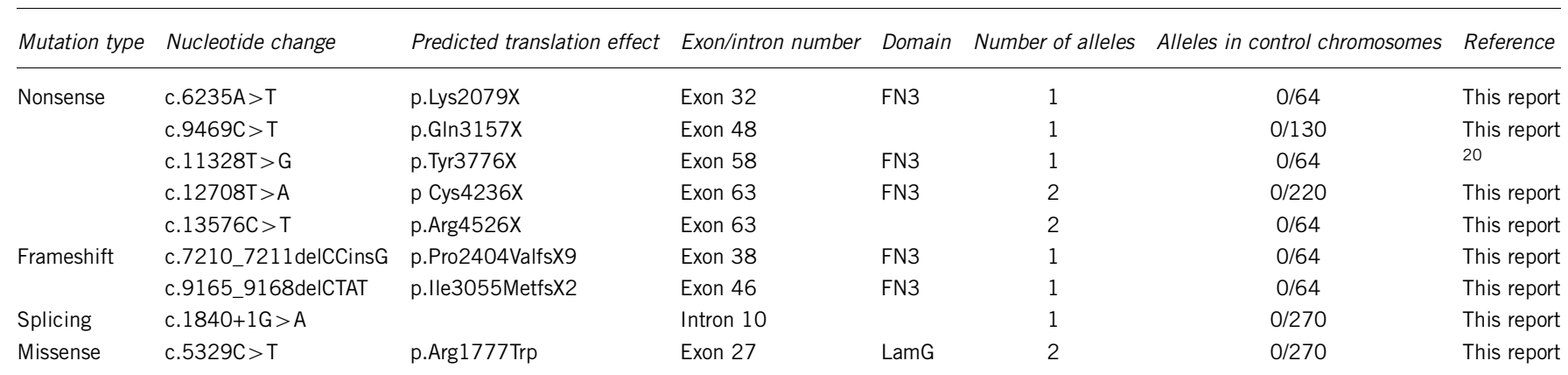

Abbreviations: FN3, fibronectin type 3 domain; LamG, laminin G domain.

Table 3 Presumed non-pathogenic alterations that have never been reported

\begin{tabular}{|c|c|c|c|c|c|}
\hline Nucleotide change & Predicted translation effect & Exon/intron number & Domain & Number of alleles & Alleles in control chromosomes \\
\hline c.785-16_785-15delAT & & Intron 4 & & 1 & $1 / 270$ \\
\hline c.5573-36delC & & Intron 27 & & 1 & $7 / 130$ \\
\hline c. $9258+15 T>C$ & & Intron 46 & & 2 & \\
\hline c. $13847 \mathrm{G}>\mathrm{T}$ & p.Gly4616Val & Exon 64 & FN3 & 2 & \\
\hline c. $14642 \mathrm{G}>\mathrm{C}$ & p.Ser4881Thr & Exon 67 & FN3 & 2 & $3 / 270$ \\
\hline
\end{tabular}

Abbreviation: FN3, fibronectin type 3 domain.

various reasons. Many of them have been reported as polymorphisms in previous reports (Supplementary Table 1). One of the two newly identified alterations within the exons (p.Ser4881Thr) was also identified in the control chromosomes. The other alteration, p.Gly4616Val, was also considered benign because the alteration was detected together with probable pathogenic mutations in patient C187. Two of the three intronic alterations that were in or close to a splicing donor site or branch point sequences (c.785-16_785-15delAT and c.5573-36delC) were also identified in the control chromosomes. As c.785-16_785-15delAT was only found in one control chromosome, RT-PCR analysis was performed to examine its precise effect on splicing. Agarose gel electrophoresis of the RT-PCR products revealed a single band of the size predicted from the normal sequence, indicating that the nucleotide change had no effect on splicing and was presumably non-pathogenic (Figure 1b).

\section{Haplotype analysis}

The c.8559-2A $>$ G splicing mutation, which we previously reported as a possible frequent Japanese mutation, was not detected in any patients in this study. To characterize the alleles, which harbor c.8559-2A $>$ G, we analyzed the haplotype pattern of 23 SNP sites (listed in Table 4) within the region surrounding the mutation site for the c.8559-2G and c.8559-2A alleles of all patients in whom at least one mutated allele was detected in this and previous reports.

In all four patients with c.8559-2A $>$ G (C152, C452, C557 and $\mathrm{C} 237$ ), the USH2A mutations were in the compound heterozygous state, as we previously reported (Supplementary Table 2). Twenty SNPs located from exon 2 to intron 61 showed the exactly same haplotype pattern for the c.8559-2G (mutated) alleles in three patients (C152, C452 and C557, Table 4). In contrast, the c.8559-2A alleles in these three patients as well as other patients without c.8559-2A $>G$ showed considerably different haplotype patterns (Table 5). As for patient C237, two heterozygous SNP sites in introns 52 and 60 could not be assigned to the c.8559-2A or c.8559-2G allele because of difficulties in obtaining family samples. However, all of the other 18
SNP patterns from exon 2 to intron 61 coincided with the c.8559-2G alleles, but not with any of the c.8559-2A alleles.

\section{Clinical findings}

All seven patients in whom at least one mutated allele was identified had developed night blindness at 10-21 years old (mean \pm s.d., $14 \pm 4.1$ years) and had been diagnosed with RP by ophthalmologists at $12-35$ years old $(27 \pm 8.2$ years, Table 1$)$. In all patients, the visual fields were symmetrically constricted, pigmentary degeneration was typical for RP with peripheral bone-spicule pigmentation and standard combined electroretinography was extinguished. The best-corrected visual acuity ranged from 1.2 to 0.06 . Three patients (C112, C552 and C185) reported having cataracts and underwent cataract surgery in both eyes.

The patients were diagnosed with hearing impairment by otorhinolaryngologists at $3-13$ years old $(6.7 \pm 3.5$ years, Table 1$)$. All patients had intelligible speech and wore hearing aids, except for patient C406. Tympanometry yielded normal results consistent with the clinical findings of a normal tympanic membrane and middle ear cavity. Audiograms showed bilateral mild-to-severe sensorineural HL with a typical slope toward high frequencies (mean level, $63.6 \pm 15.6 \mathrm{~dB})$.

A delay in motor development was not reported, and all patients started walking before they were 18 months old. The caloric test was normal among all the patients on whom the test was performed; the caloric test was not conducted on patient C187 (Table 1). These results indicate that all patients, except for patient C187, had normal vestibular function, although additional evaluations (for example, the rotary chair test) with potentially greater sensitivity for detecting subtle vestibular dysfunction were not performed.

\section{DISCUSSION}

We previously described 14 USH2A mutations from 10 Japanese USH2 patients, of which 11 were novel. ${ }^{22}$ In this study, we identified nine different mutations from nine patients, of which eight were 
Table 4 USH2A haplotype patterns of c.8559-2G alleles (genotypes of the patients are shown in Supplementary Table 2)

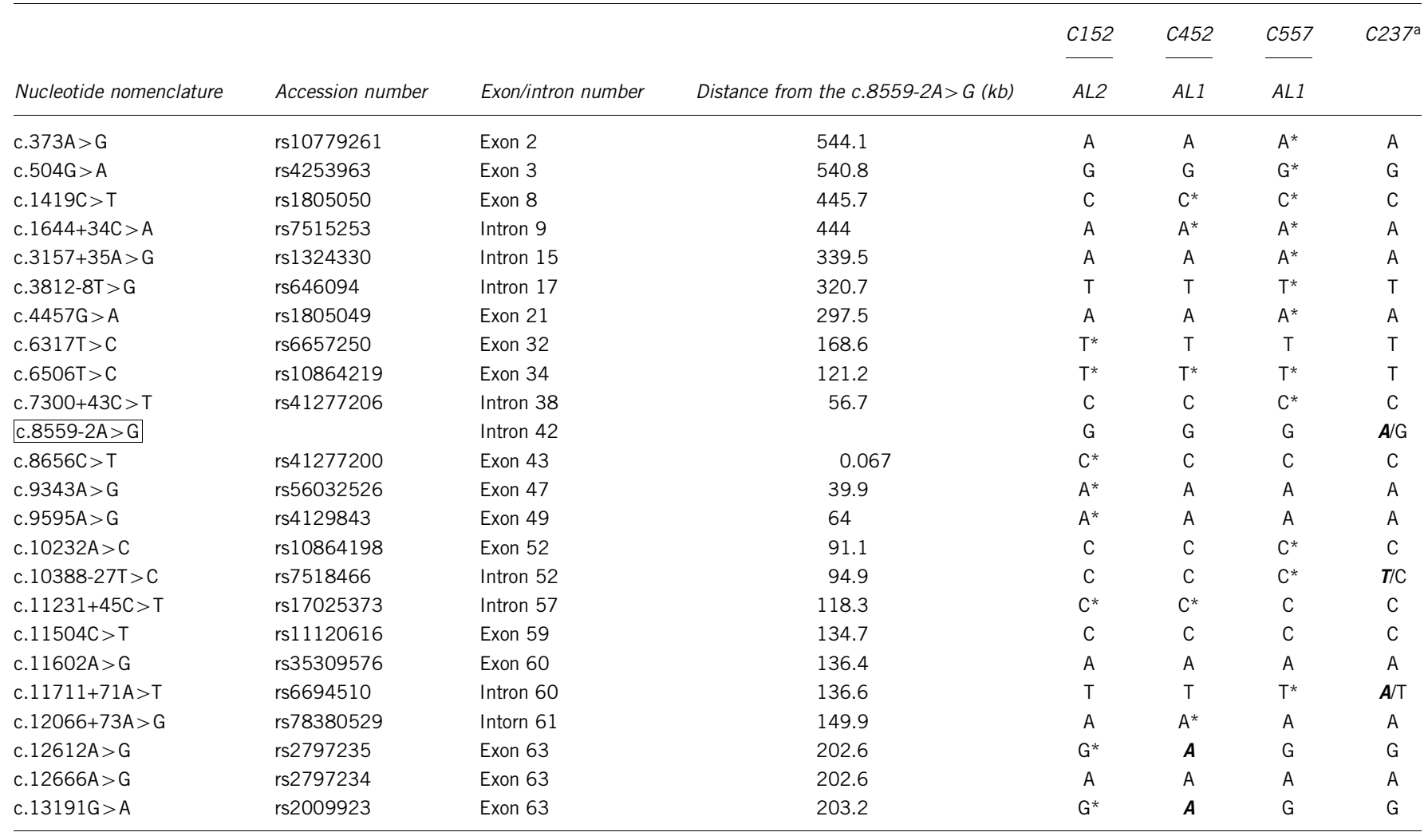

Abbreviation: AL, allele.

Nucleotides described in bold italic style denote that they are different from the others.

Asterisks above nucleotides indicate that segregation analysis was performed to determine which of the two nucleotides exist on the c.8559-2G allele.

aFor patient C237, segregation analysis was not performed because samples from family members were not available.

novel. As one of these mutations had been previously identified in Scandinavian patients, ${ }^{20}$ all of the mutations found in this study were different from the 14 mutations we previously identified. In total, we have identified 23 mutations in 15 of 19 patients, of which 19 were novel. Four mutations, which had been identified in non-Japanese populations, did not include p.Glu767fs, the most prevalent mutation in Caucasians that accounts for $\sim 30 \%$ of mutated alleles. ${ }^{18-21}$ These results indicate that the mutation spectrum for USH2A among Japanese patients largely differs from Caucasian, Jewish and Palestinian patients. ${ }^{12,18-21,26,29-32}$ In spite of this difference, the frequency of Japanese patients carrying USH2A mutations in USH2 (79\%) is similar to that of the above-mentioned populations. ${ }^{18-20,30,32}$ For these reasons, mutation screening for USH2A is a highly sensitive method for diagnosing USH2, but mutation screening for the p.Glu767fs mutations is not effective among Japanese patients.

In this study, we did not identify the c.8559-2A $>\mathrm{G}$ splicing mutation, which we previously reported as a possible frequent mutation in Japanese USH2 patients. SNP analysis for the region surrounding c.8559-2 for the c.8559-2G (mutated) and c.8559-2A (nonmutated) alleles was performed on 15 patients. For the c.8559-2G alleles, we identified the same haplotype pattern over a long range from exon 2 to exon 52 (at least $635 \mathrm{~kb}$ ) in all four c.8559-2A>G patients; however we did not observe such a haplotype pattern for the c.8559-2A alleles. This indicates that the $635-\mathrm{kb}$ region including c.8559-2A $>\mathrm{G}$ is in linkage disequilibrium and strongly suggests that the mutation originated from a common ancestor. In three patients, except for patient C237, the common haplotype region was even longer (at least $694 \mathrm{~kb}$ from exon 2 to intron 61). It is quite possible that the c.8559-2G allele of patient C237 also has the same haplotype pattern with that of the other three patients from exon 2 to intron 61 ; however, we could not examine this in this patient. If that is the case, the 694-kb linkage disequilibrium region is common in all of the c.8559-2G alleles in this study.

It may be worth describing that the four c.8559-2A $>\mathrm{G}$ patients were born in western Japan. A possible reason why we did not find the splicing mutation in this study may be that the nine patients analyzed in the present study are mostly from eastern Japan (Figure 1c). Even though the number of patients studied was small, these findings may suggest that the mutation is distributed mainly in western Japan. The fact that c.8559-2A $>\mathrm{G}$ was also detected in two Chinese patients suggests the possibility that the mutation occurred in an ancient common ancestor. ${ }^{33}$ Further analysis is necessary to obtain a more precise mutation spectrum of $U S H 2 A$ in the Japanese.

In conclusion, mutation screening of USH $2 A$ elucidated nine mutations in seven of nine patients, confirming that mutation screening of USH2A is effective for the early diagnosis of USH2 and the mutation spectrum of Japanese patients differs from the spectra of various ethnicities, including Caucasian, Jewish and Palestinian. Haplotype analysis of the c.8559-2A $>\mathrm{G}$ allele indicated that it originated from an ancestral mutational event and the mutation was likely to be distributed mainly in western Japan. Most of the patients involved in this study came from eastern Japan, which contributed to the absence of c.8559-2A $>\mathrm{G}$ in this study. Further analysis is necessary to obtain a more precise mutation spectrum of $U S H 2 A$ in the Japanese. 
Table 5 USH2A haplotype patterns of c.8559-2A alleles (Genotypes of the patients are shown in Table 1 and Supplementary Table 2)

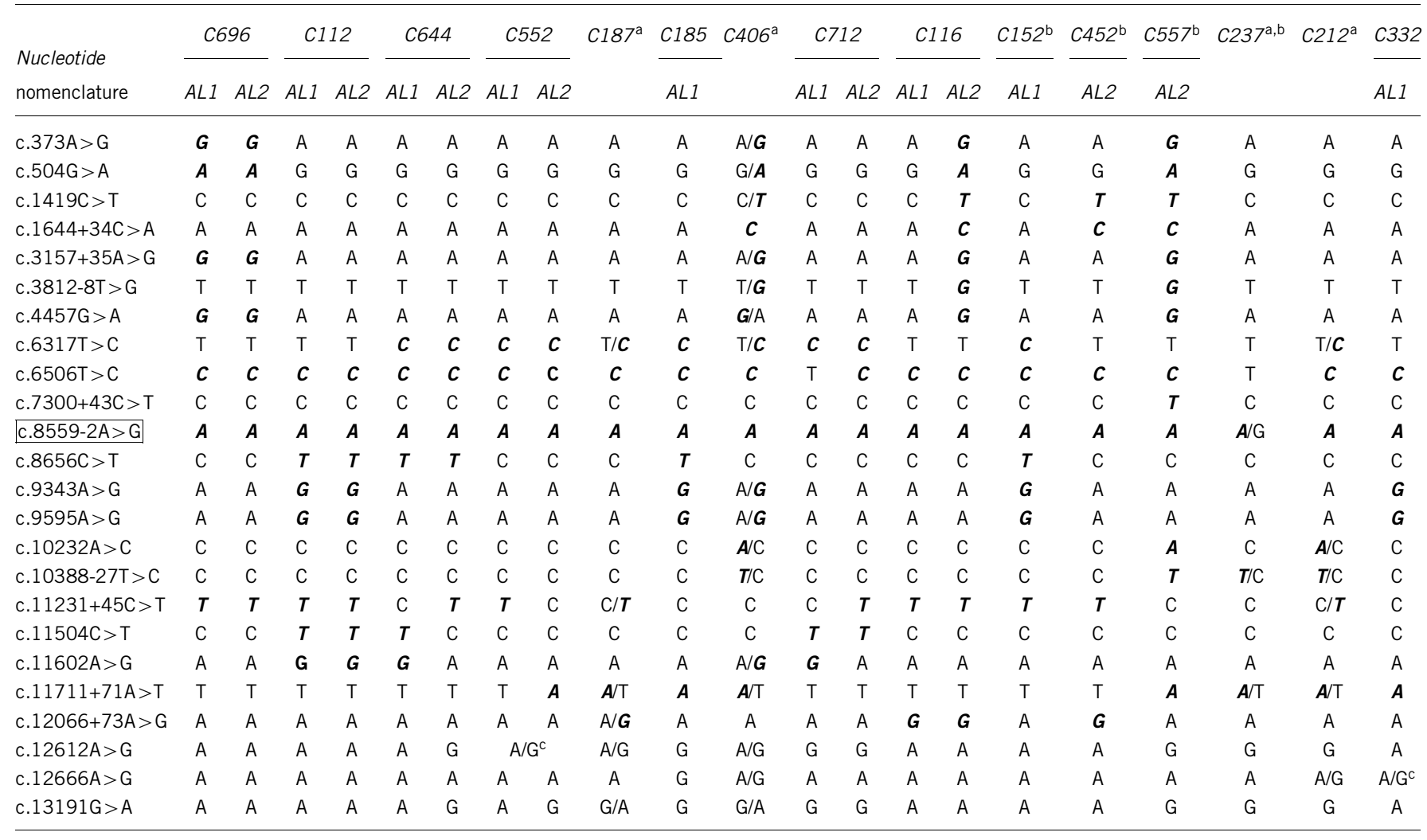

Abbreviation: AL, allele.

Nucleotides described in bold italic style denotes that they are different from that of c.8559-2G alleles ranging from c.373A $>$ G to c.12066+73A $>$ G shown in Table 4 .

aFor four patients (C187, C406, C237 and C212), segregation analysis was not performed because samples from family members were not available.

bFor four patients (C152, C452, C557 and C237), the haplotypes for c.8559-2G alleles are shown in Table 4.

${ }^{c}$ For two single nucleotide polymorphisms (c.12612A $>$ G on patient C552 and c.12666A $>$ G on patient C332), genotyping was incomplete because both of the parents also had A/G alleles.

\section{ACKNOWLEDGEMENTS}

We thank all the subjects who participated in this study. This work was supported by research grants from the Ministry of Labor and Welfare (Acute Profound Deafness Research Committee) and the Ministry of Education, Culture, Sports, Science, and Technology (Young Scientists Grant B-22791589) in Japan.

1 Yan, D. \& Liu, X. Z. Genetics and pathological mechanisms of Usher syndrome. J. Hum. Genet. 55, 327-335 (2010).

2 Nuutila, A. Dystrophia retinae pigmentosa-dysacusis syndrome (DRD): a study of the Usher or Hallgren syndrome. J. Genet. Hum. 18, 57-88 (1970).

3 Boughman, J. A., Vernon, M. \& Shaver, K. A. Usher syndrome: definition and estimate of prevalence from two high-risk populations. J. Chronic Dis. 36, 595-603 (1983).

4 Grondahl, J. Estimation of prognosis and prevalence of retinitis pigmentosa and Usher syndrome in Norway. Clin. Genet. 31, 255-264 (1987).

5 Hope, C. I., Bundey, S., Proops, D. \& Fielder, A. R. Usher syndrome in the city of Birmingham: prevalence and clinical classification. Br. J. Ophthalmol. 81, 46-53 (1997).

6 Rosenberg, T., Haim, M., Hauch, A- M. \& Parving, A. The prevalence of Usher syndrome and other retinal dystrophy-hearing impairment associations. Clin. Genet. 51, 314-321 (1997).

7 Spandau, U. H. \& Rohrschneider, K. Prevalence and geographical distribution of Usher syndrome in Germany. Graefes Arch. Clin. Exp. Ophthalmol. 240, 495-498 (2002).

8 Kimberling, W. J. \& Möller, C. Clinical and molecular genetics of Usher syndrome. J. Am. Acad. Audiol. 6, 63-72 (1995).

9 Tsilou, E. T., Rubin, B. I., Caruso, R. C., Reed, G. F., Pikus, A., Hejtmancik, J. F. et al. Usher syndrome clinical types I and II: could ocular symptoms and signs differentiate between the two types? Acta. Ophthalmol. Scand. 80, 196-201 (2002).
10 Pennings, R. J. E., Huygen, P. L. M., Orten, D. J., Wagenaar, M., van Aarem, A., Kremer, $\mathrm{H}$. et al. Evaluation of visual impairment in Usher syndrome $1 \mathrm{~b}$ and Usher syndrome 2a. Acta. Ophthalmol. Scand. 82, 131-139 (2004).

11 Eudy, J. D., Weston, M. D., Yao, S., Hoover, D. M., Rehm, H. L., Ma-Edmonds, M. et al. Mutation of a gene encoding a protein with extracellular matrix motifs in Usher syndrome type Ila. Science 280, 1753-1757 (1998).

12 van Wijk, E., Pennings, R. J., te Brinke, H., Claassen, A., Yntema, H. G., Hoefsloot, L. $\mathrm{H}$. et al. Identification of 51 novel exons of the Usher syndrome type 2A (USH2A) gene that encode multiple conserved functional domains and that are mutated in patients with Usher syndrome type II. Am. J. Hum. Genet. 74, 738-744 (2004).

13 Weston, M. D., Luijendijk, M. W., Humphrey, K. D., Moller, C. \& Kimberling, W. J. Mutations in the VLGR1 gene implicate G-protein signaling in the pathogenesis of Usher syndrome type II. Am. J. Hum. Genet. 74, 357-366 (2004).

14 Ebermann, I., Scholl, H. P., Charbel Issa, P., Becirovic, E., Lamprecht, J., Jurklies, B. et al. A novel gene for Usher syndrome type 2: mutations in the long isoform of whirlin are associated with retinitis pigmentosa and sensorineural hearing loss. Hum. Genet. 121, 203-211 (2007).

15 Weston, M. D., Eudy, J. D., Fujita, S., Yao, S., Usami, S., Cremers, C. et al. Genomic structure and identification of novel mutations in usherin, the gene responsible for Usher syndrome type Ila. Am. J. Hum. Genet. 66, 1199-1210 (2000).

16 Dreyer, B., Tranebjaerg, L., Brox, V., Rosenberg, T., Moller, C., Beneyto, M. et al. A common ancestral origin of the frequent and widespread 2299delG USH2A mutation. Am. J. Hum. Genet. 69, 228-234 (2001).

17 Pennings, R. J., Te Brinke, H., Weston, M. D., Claassen, A., Orten, D. J., Weekamp, H. et al. USH2A mutation analysis in 70 Dutch families with Usher syndrome type II. Hum. Mutat. 24, 185 (2004).

18 Aller, E., Jaijo, T., Beneyto, M., Najera, C., Oltra, S., Ayuso, C. et al. Identification of 14 novel mutations in the long isoform of USH2A in Spanish patients with Usher syndrome type II. J. Med. Genet. 43, e55 (2006).

19 Baux, D., Larrieu, L., Blanchet, C., Hamel, C., Ben Salah, S., Vielle, A. et al. Molecular and in silico analyses of the full-length isoform of usherin identify new pathogenic alleles in Usher type II patients. Hum. Mutat. 28, 781-789 (2007).

20 Dreyer, B., Brox, V., Tranebjaerg, L., Rosenberg, T., Sadeghi, A. M., Moller, C. et al. Spectrum of USH2A mutations in Scandinavian patients with Usher syndrome type II. Hum. Mutat. 29, 451 (2008). 
21 Yan, D., Ouyang, X., Patterson, D. M., Du, L. L., Jacobson, S. G. \& Liu, X. Z. Mutation analysis in the long isoform of USH2A in American patients with Usher syndrome type II. J. Hum. Genet. 54, 732-738 (2009).

22 Nakanishi, H., Ohtsubo, M., Iwasaki, S., Hotta, Y., Mizuta, K., Mineta, H. et al. Identification of 11 novel mutations in USH2A among Japanese patients with Usher syndrome type 2. Clin. Genet. 76, 383-391 (2009).

23 Nakanishi, H., Ohtsubo, M., Iwasaki, S., Hotta, Y., Takizawa, Y., Hosono, K. et al. Mutation analysis of the MYO7A and $\mathrm{CDH} 23$ genes in Japanese patients with Usher syndrome type 1. J. Hum. Genet. 55, 796-800 (2010).

$24 \mathrm{Ng}$, P. C. \& Henikoff, S. Predicting deleterious amino acid substitutions. Genome Res. 11, 863-874 (2001).

25 Ramensky, V., Bork, P. \& Sunyaev, S. Human non-synonymous SNPs: server and survey. Nucleic Acids Res. 30, 3894-3900 (2002).

26 McGee, T. L., Seyedahmadi, B. J., Sweeney, M. O., Dryja, T. P. \& Berson, E. L. Novel mutations in the long isoform of the USH2A gene in patients with Usher syndrome type II or non-syndromic retinitis pigmentosa. J. Med. Genet. 47, 499-506 (2010).

27 Nakanishi, H., Ohtsubo, M., Iwasaki, S., Hotta, Y., Mizuta, K., Mineta, H. et al. Hair roots as an mRNA source for mutation analysis of Usher-syndrome causing genes. J. Hum. Genet. 55, 701-703 (2010).
28 Dreyer, B., Tranebjaerg, L., Rosenberg, T., Weston, M. D., Kimberling, W. J. \& Nilssen, 0 . Identification of novel USH2A mutations: implications for the structure of USH2A protein. Eur. J. Hum. Genet. 8, 500-506 (2000).

29 Kaiserman, N., Obolensky, A., Banin, E. \& Sharon, D. Novel USH2A mutations in Israeli patients with retinitis pigmentosa and Usher syndrome type 2. Arch. Ophthalmol. 125, 219-224 (2007).

30 Auslender, N., Bandah, D., Rizel, L., Behar, D. M., Shohat, M., Banin, E. et al. Four USH2A founder mutations underlie the majority of Usher syndrome type 2 cases among non-Ashkenazi Jews. Genet. Test 12, 289-294 (2008).

31 Sandberg, M. A., Rosner, B., Weigel-DiFranco, C., McGee, T. L., Dryja, T. P. \& Berson, E. L. Disease course in patients with autosomal recessive retinitis pigmentosa due to the USH2A gene. Invest. Ophthalmol. Vis. Sci. 49, 5532-5539 (2008).

32 Ebermann, I., Koenekoop, R. K., Lopez, I., Bou-Khzam, L., Pigeon, R. \& Bolz, H. J. An USH2A founder mutation is the major cause of Usher syndrome type 2 in Canadians of French origin and confirms common roots of Quebecois and Acadians. Eur. J. Hum. Genet. 17, 80-84 (2009).

33 Dai, H., Zhang, X., Zhao, X., Deng, T., Dong, B., Wang, J. et al. Identification of five novel mutations in the long isoform of the USH2A gene in Chinese families with Usher syndrome type II. Mol. Vis. 14, 2067-2075 (2008).

Supplementary Information accompanies the paper on Journal of Human Genetics website (http://www.nature.com/jhg) 